\title{
Mathematics for the Modeling of Defects in Materials
}

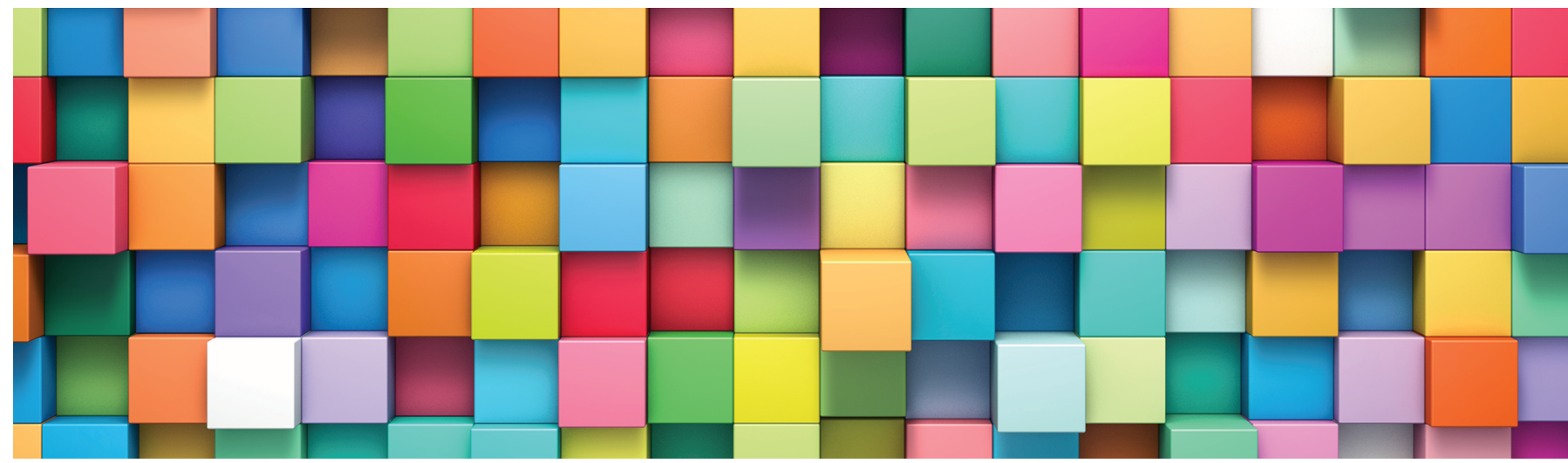

\section{Claude Le Bris}

\section{Introduction}

A famous quote ${ }^{1}$ says that "Crystals are like people, it is the defects in them which tend to make them interesting." One of the best known examples of the importance of defects in materials is perhaps the fact that crystals, and more generally materials in an ordered phase (as opposed to disordered or amorphous materials such as glasses), owe their plasticity to the existence and motion of dislocations, which are linear topological defects associated with an elastic stress field-a line of miscoordinated atomic sites indicating an irregularity within the periodic array of atoms (see Figure 1). As the stress increases and exceeds a certain threshold, the deformation is no longer reversible, as is the case in the elastic regime. The displacement of dislocations allows the material still to deform, but in a nonelastic, nonreversible manner. The density, the topology, the possible entanglements of these defects control this plastic behavior, in sharp contrast with the reversible, elastic behavior that is ruled by the geometry of the underlying, idealistically perfectly periodic, atomistic structure. Plasticity explains why one is able to shape metallic materials and

Claude Le Bris is a researcher at Ecole des Ponts and Inria, Paris, France. His email address is claude.1e-bris@enpc.fr.

The research of the author is partially supported by ONR under Grant N0001415-1-2777, and by EOARD under Grant FA-9550-17-1-0294.

${ }^{1}$ C. J. Humphreys, Stem Imaging of Crystals and Defects, in Introduction to Analytical Electron Microscopy, pp. 305-332, J. J. Hren et al. (eds.), 1979. Communicated by Notices Associate Editor Reza Malek-Madani.

For permission to reprint this article, please contact:

reprint-permission@ams.org.

DOI: https://doi.org/10.1090/noti2098 to manufacture tools. Without these mobile defects, materials would deform elastically until they suddenly break, and bending even the thinnest metal bar would require a large amount of energy. Likewise, in a different domain, doped semiconductors, and therefore electronics, would not exist without defects. Defects are useful for something!

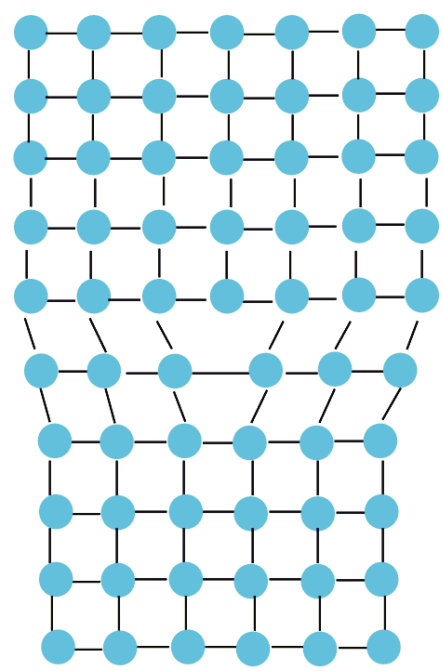

Figure 1. Simple-called edge-dislocation at the microscopic scale: an extra half-plane of atoms, the "defects," is inserted in the periodic lattice (2-dimensional cut of a structure periodic in the perpendicular direction).

More generally, defects in media, not only materials, play a key practical role in several applications. One may have in mind all applications of computer vision and imaging that aim to detect an unusual feature within an image and, 
beyond, the many applications in inverse problems in the engineering sciences-localization of an oil reservoir in the underground, radars, sonars, etc. Interestingly, the inverse problem of detecting defects is also relevant in the context of materials, for instance for crack detection in metals. However, before addressing the inverse problem, one first needs to adequately formalize the direct problem: the modeling and study of defects are already critical issues for materials.

In a nutshell, one may see a defect as a presumably rare anomaly within an otherwise well-understood and often homogeneous background. It would be impossible to cover the huge variety of defects in materials science; we refer to the many excellent monographs in mechanicsrelated fields. The purpose of this mathematically oriented presentation is to show a specific series of settings in materials science, and more precisely multi-scale materials science, where mathematics plays a role in the modeling and thus in the understanding and numerical approximation of defects. Even though our study can be motivated by practical concerns such as the modeling of plasticity, which we mentioned at the opening of this article, we mostly consider here defects that are much simpler than dislocations and we consider them as mathematical objects independently of the meaning they can carry in a mechanical context. Our purpose is also to present a set of theoretical questions that, conversely, arise from this particular class of applied problems. In the absence of defects, the mathematical problem is "nice and easy" - by the standards of contemporary functional and differential equations analysis. It derives from well-established techniques. It is also expected to be simple computationally. The presence of a defect breaks this nice mathematical setting. The mathematical difficulty is due to the ensuing losses of finiteness, boundedness, or compactness. Numerical difficulties similarly follow. New tools are everywhere in order.

Of course, this article cannot cover the many existing mathematical studies, in various areas of mathematics, that address the modeling of defects. It only presents an expository review of our approach to the problem, in collaboration with close colleagues and focused on the theory of partial differential equations and related issues, along with connections with some other works that we find particularly intimately related to ours.

\section{Atomistic Models and Beyond}

A simple atomistic model. To begin with, we consider a simplistic, 1-dimensional model of a material-at zero temperature, with only one atomic species-and we introduce the notion of defect in an atomic lattice at an elementary level. A set of $2 N+1$, eventually $N=\infty$, point particles or atoms $X_{k}, k \in\{-N, \ldots, N\}$, with the convention $X_{-N} \leq$ $X_{-N+1} \leq \cdots \leq X_{N}$ interact via a nearest-neighbor pair interaction potential $V$. By this we mean that a given particle in $X_{k}$ only interacts with the particles $X_{k-1}$ and $X_{k+1}$ (with the obvious adaptation when $k=-N$ or $N$ ). When $N$ is finite, as in a finite size molecular system, the energy of the system is the sum of all interactions:

$$
E_{N}=\sum_{-N \leq k \leq N-1} V\left(\left|X_{k+1}-X_{k}\right|\right) .
$$

Suppose now that the particles are clamped at integer positions, $X_{k}=k$, which is typically the case if the pair interaction potential $V(r)$, which is assumed to vanish at infinity, achieves its unique (negative) minimal value at $r=1$ and one considers the equilibrium, lowest energy, configuration of the set of $2 N+1$ particles. Next let $N$ grow to infinity. The energy $E_{N}=2 N V(1)$ clearly diverges. This is not unexpected, since the energy is an extensive quantity that scales linearly with respect to the amount of matter considered. Only the energy per particle admits a limit, namely

$$
\frac{1}{2 N+1} E_{N} \longrightarrow V(1)
$$

as $N \rightarrow+\infty$. The latter value defines the energy, in this model, of the periodic system consisting of an infinity of periodically arranged atoms $X_{k}=k, k \in \mathbb{Z}$. Let us now present two ways to perturb this ideal periodic system.

First, we may modify the positions of all particles. This may be achieved assuming now that the $k$-th particle has a random position $X_{k}(\omega)=k+Y_{k}(\omega)$, slightly shifted from $k$, where, say, all the random variables $Y_{k},-N \leq$ $k \leq N$, are independent, identically distributed, and valued in $(-1 / 2,1 / 2)$. A similar argument as above, this time formalized by the law of large numbers, yields the expectation value $\mathbb{E}\left(V\left(1+Y_{1}-Y_{0}\right)\right)$ as the energy per particle of the infinite system we have constructed. We may also modify the positions of particles deterministically: for instance, the system

$$
X_{k}= \begin{cases}k+1 / 2 & \text { for }(2 n)^{2}<|k| \leq(2 n+1)^{2}, \\ k & \text { for }(2 n+1)^{2}<|k| \leq(2 n+2)^{2},\end{cases}
$$

composed of periodic regions alternately shifted from $1 / 2$ or not, clearly admits an energy per particle-which, incidentally, is identical to that of the original periodic system-even though the system is by no means periodic and the support of the defects extends to infinity. In other terms, the defect is global, as in the random case, although entirely deterministic.

Another direction we may take is to leave all the particles unperturbed except only a few, say one, namely the particle $k=0$, which we now clamp at $X_{0}=a \neq 0$. This is now our notion of local defect. The energy of the finite 
system now reads as

$$
\begin{aligned}
E_{N}^{\text {defect }}= & \sum_{k=-N}^{-2} V\left(\left|X_{k+1}-X_{k}\right|\right) \\
& +V(|1+a|)+V(|1-a|) \\
& +\sum_{k=1}^{N-1} V\left(\left|X_{k+1}-X_{k}\right|\right) .
\end{aligned}
$$

Of course, $\frac{1}{2 N+1} E_{N}^{\text {defect }}$ shares the same limit as $\frac{1}{2 N+1} E_{N}$ when $N$ grows to infinity. Our defect being localized, it does not affect the energy per particle in the limit of an infinite number of particles. In order to get a nontrivial effect-and "detect" the defect simply because the energy of the system differs from that of the periodic system-we have to proceed otherwise. To model the energy of the perturbed system differently, we consider the difference

$$
E_{N}^{\text {defect }}-E_{N}=V(|1+a|)+V(|1-a|)-2 V(1),
$$

which defines a quantity (the defect formation energy, in some sense) that, contrary to either of the two terms of the left-hand side taken separately, has a finite limit when $N \rightarrow$ $+\infty$. Clearly, this limit may be seen as the energy of the periodic system with a defect at zero, being understood that this energy is counted with reference to the ideal periodic system.

Beyond atomistics. The above simplistic model can be made more sophisticated in several directions. One may consider an analogous discrete system of infinitely many atomic sites, in the ambiant 3-dimensional physical space, all interacting with one another via a potential. Think for instance of an interaction potential $V \in L^{1}\left(\mathbb{R}^{n}\right) \cap$ $C^{0}\left(\mathbb{R}^{n} \backslash\{0\}\right)$, and it is then an easy exercise to generalize the formulae above and discover that the corresponding periodic potential $V_{\text {per }}=\sum_{k \in \mathbb{Z}^{n}} V(\cdot-k)$ plays a key role. Such discrete models offer many cases of specific interest.

On the theoretical and modeling fronts, they can serve as a starting point for a change of scale and the derivation of models of continuum mechanics $[9,10]$. Many of the latter models are indeed phenomenological, not to say ad hoc. Their mathematical study is a challenge, in particular when the material has indeed defects. As an example, one may think of the models of fracture and the works by Gilles Francfort and his collaborators. The ab initio derivation of continuum models has been completed for some models, for instance in elasticity, with various approaches and in particular $\Gamma$-limit techniques-works by Andrea Braides and his collaborators; see [6] for a survey. Large-scale limits of dislocation dynamics, sometimes using homogenization theory, have also been investigated. The range of macroscopic models that can be justified by such an ab initio derivation is however limited. In particular, in relation to our earlier discussion and in line with several existing works, it would be of major theoretical and practical interest to better understand, from models of defects at the atomistic scale, the often phenomenological models of plasticity.

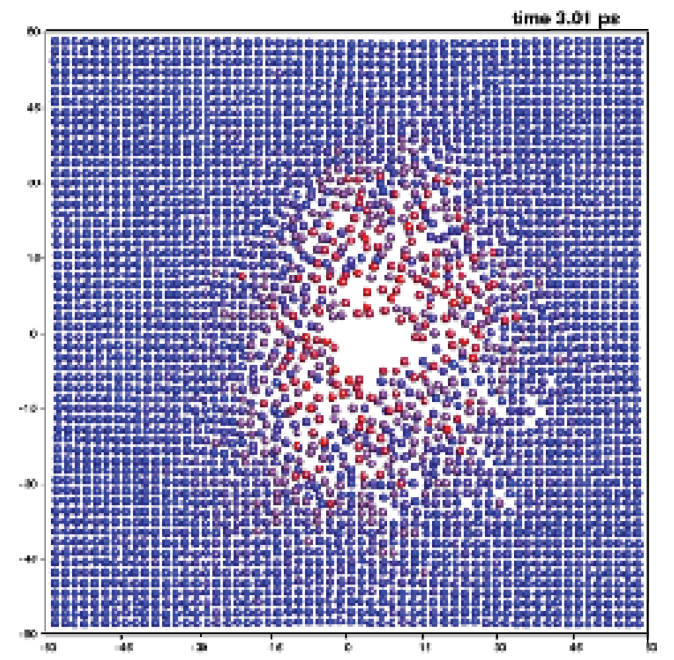

Figure 2. Computer simulation of a displacement cascade.

In numerical simulations, atomistic models, possibly embedding defects, can be used to better simulate the behavior of real materials. An illustrative case is provided by the context of nuclear engineering. The aging of materials is studied by successively following in time (a) the evolution of irradiation defects in the periodic structure-the displacement cascades of Figure 2, (b) the aggregation of defects to form dislocations, (c) the evolution of dislocations, and eventually (d) the effective dynamics of the density of the different categories of dislocations. Each stage in this string of modeling levels is a coarse-grained version of its predecessor. The defects, originally treated as discrete microscopic objects, are monitored, at the macroscopic level, as a density field of objects in interaction which move and transform. These techniques belong to the vast category of molecular dynamics techniques-the "molecule" being, in this instance, the defects-although a more accurate characterization would be computational statistical mechanics techniques; see e.g. [17]. A range of stochastic numerical techniques is employed to achieve this, which of course concurrently raises a series of interesting mathematical questions. The simulation of metastable trajectories and rare events are the central mathematical problematics of the field.

Atomistic models are also used in practice in computational multi-scale mechanics. Some approaches-an example of which is the so-called quasi-continuum methodindeed use different models, either continuous or discrete, 


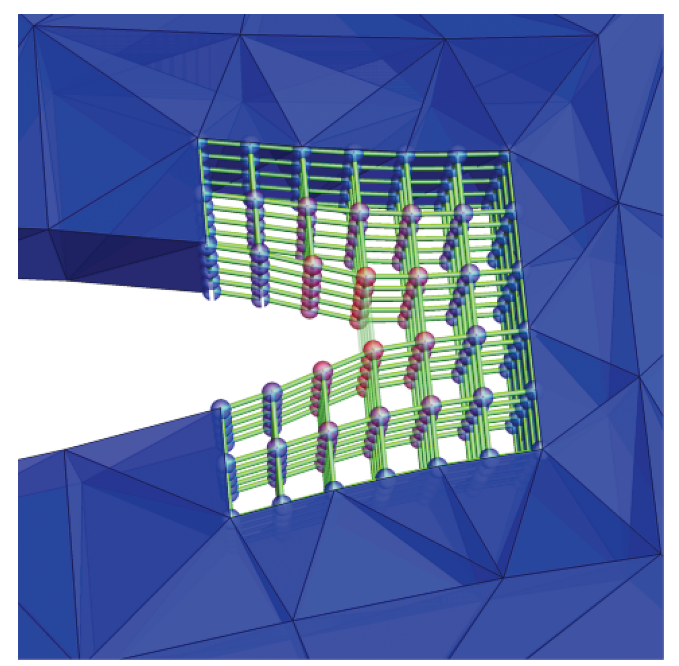

Figure 3. Atomistic-to-Continuum simulation of a material. Microcrack opening in a periodic lattice: at the vicinity of the fracture, where defects may appear and propagate, the description is atomistic. Elsewhere, it is based on the continuum approximation. The two levels of description are coupled seamlessly.

in different regions of the material. The former category of models are-a priori or adaptively-selected in the regions of the material where the strains and stresses are expected to vary smoothly at the atomistic scale, while the latter category of models are restricted to pathological regions, typically containing atomistic defects of the crystalline structure created by the large variations of stresses and strains over atomistic distances; see Figure 3 for an illustration. The issues discussed above lay the groundwork for the numerical analysis of such simulation approaches. The works of Mitchell Luskin, Christoph Ortner, and their collaborators give more details on the mathematical understanding, the numerical analysis, and the latest developments of the approach; see [18].

An alternate direction-and eventually a complementary direction when all levels of modeling are combined together-can be to consider the microscopic scale only, but refine the atomistic model using a model that accounts for the electronic structure of the material. Put differently, classical mechanics is replaced by quantum mechanics. Models of the crystalline phase, used in practice in computational solid state physics, can typically be obtained by a bulk limit process as above. Density functional theory type models-Thomas-Fermi, orbitalfree, Kohn-Sham-type models; wave-functions modelsHartree, Hartree-Fock type models; or even more sophisticated models, can all be derived in this manner. Mathematically, the problem can be stated as identifying the limit of the energy per unit volume, namely the analogous quantity to (2) suitably rescaled. The energy $E_{N}$ is now a minimization problem set on functions, e.g. the density $\rho$, describing the electronic state and parametrically depending on the parameter $N$ that, up to mathematically irrelevant technicalities, fixes the total nuclear charge of the nuclei located at the points $X_{k}$ and the total electronic charge:

$$
E_{N}=\inf \left\{\mathcal{E}\left(\rho,\left\{X_{k}\right\}\right) ; \rho \geq 0 \int_{\mathbb{R}^{n}} \rho=N\right\} .
$$

The answer to this question is already challenging for periodic geometries in the absence of defects and may require subtracting the energy of a reference infinite system to make the limit finite, exactly as we did above for our simplistic atomistic system. A typical reference system popular in solid state physics is a jellium, that is, a homogeneously charged background. Proving that models for finite-size microscopic systems have a large size $(N \rightarrow+\infty)$ limit that coincides with models for the condensed phase is an important mathematical endeavor, with several outstanding contributions by Charles Fefferman, Elliott Lieb, Barry Simon, etc. The question, known as the bulk or thermodynamic limit problem, has been examined for models at zero, or at positive temperature, for models that are either classical or quantum in nature, etc. We refer to [16] for a survey.

Understanding how defects affect this process, and accounting for them, is an additional layer of difficulty, which has only been attempted for some models, and for which various techniques of renormalization are useful. State-of-the-art works in this direction include works by Eric Cancès [7], Jianfeng Lu, Mathieu Lewin, and their respective collaborators.

The rationale. Although extremely, not to say overly, simplified, the above elementary atomistic model allows us to illustrate some key difficulties related to the modeling of defects. In the absence of defects, we have a gentle situation, where a geometric assumption, here periodicity, followed by a natural technique, here division of the energy by the number of particles, allow us to model the infinite system as a finite one. Put differently, a problem a priori set on the-noncompact-whole space "miraculously" reduces to a problem on a unit cell. Formerly, possible difficulties were expected to prove the existence of minimizers, define the set of admissible functions-position, displacement, etc.-depending on their decay at infinity. In contrast, every manipulation is now expected to be simple, and a setting using only classical functional spaces and compactness techniques is sufficient. This in turn allows us to anticipate, on the applied front, the use of standard discretization and numerical simulation techniques.

When a defect is inserted, this idealistic landscape vanishes, and we observe two phenomena. Firstly, depending on the nature of the defect-whether it is localized, that is, in some vague sense integrable, or it is globalthere may be, or not, a nontrivial effect on the definition 
of the average-here, the energy per unit particle, say. In any event, deciding whether there is or is not such an effect requires us to a priori consider infinite systems that cannot be reduced to finite size systems as before. Secondly, in the case where there is no global effect, there might be a local effect that can only be seen by zooming in locally, or by subtracting the background, both techniques amounting to eliminating the problem at infinity_far away from the defect.

Note that the endeavor is subtle. On the one hand, letting the number $N$ of particles go to infinity is a first step in changing the scale at which materials are examined. In doing so, only phenomena that repeat themselves are supposed to survive. Small deviations from the average are meant to disappear. The point of modeling at a given scale is to keep from the finer scales only average information and neglect everything else that does not percolate to the scale under consideration. The reason is that most macroscopic phenomena are results of ensemble effects at a finer scale, not individual effects. On the other hand, not all macroscopic phenomena are ensemble or macroscopic effects. It is well known that the color of most gemstones is due to electronic bound states carried by impurities, a microscopic effect, which percolates throughout the scales and never gets "averaged". Likewise, we have seen that plasticity originates from defects. In sharp contrast to ensemble averaging, accounting for the presence of these small scales or these defects precisely requires that we not omit the details.

\section{Periodic Homogenization}

Our next step is to move from a discrete description of a material to a continuous description while keeping our attention focused on defects. Let us now change the setting and consider partial differential equations. For simplicity again, we begin our discussion with the diffusion equation

$$
-\operatorname{div}\left(a_{\varepsilon} \nabla u_{\varepsilon}\right)=f,
$$

which, set on a domain $\Omega \subset \mathbb{R}^{n}$ and supplied with suitable boundary conditions on $\partial \Omega$, models a large variety of physical phenomena. For example, if the coefficient $a_{\varepsilon}$ denotes the heat conductivity of the medium, and $f$ models the heat source, then the solution $u_{\varepsilon}$ is thought of as the temperature field within the domain $\Omega$. Equivalently, with vector-valued solutions $u_{\varepsilon}$ and loadings $f$, the same equation models the linear elasticity of a material that has elasticity tensor $a_{\varepsilon}$. In our notation, fields carry a subscript $\varepsilon$ to indicate that they presumably vary at a tiny lengthscale $\varepsilon$ much smaller than the typical scale of the domain $\Omega$. The question, similar to that of considering $N \rightarrow \infty$ in the previous section, concerns the behavior of the solution $u_{\varepsilon}$ in the asymptotic limit $\varepsilon \rightarrow 0$. Taking the limit aims at capturing the average behavior of $u_{\varepsilon}$, possibly within a certain level of details-meaning in various functional spacesand characterizing it, exactly as the purpose of the limit performed in the previous section is to obtain the bulk behavior of the system starting from its atomic description. Homogenization theory formalizes this question. In the prototypical and historical case where $a_{\varepsilon}=a_{\text {per }}(x / \varepsilon)$ with $a_{\text {per }}$ a periodic function at scale one, the limit $u^{*}$ of $u_{\varepsilon}$ is the solution of the homogenized equation

$$
-\operatorname{div}\left(A^{*} \nabla u^{*}\right)=f,
$$

where the homogenized coefficient $A^{*}$ can indeed be computed. The approximation of $u_{\varepsilon}$ by $u^{*}$ holds strongly in the $L^{2}(\Omega)$ sense and may be refined in the $H^{1}(\Omega)$ norm upon using a periodic corrector, which adds to $u^{*}$ the prototypical oscillation of the original problem. This corrector, or more precisely this $n$-tuple of correctors, is the solution to the periodic problem

$$
-\operatorname{div}\left(a_{\text {per }}\left(e_{i}+\nabla w_{\text {per }, i}\right)\right)=0
$$

for $1 \leq i \leq d$ and $e_{i}$ the corresponding canonical basis vector of $\mathbb{R}^{n}$. It is enlightening to realize that equation (7), set on the periodic cell of the periodic microstructure of the material encoded in $a_{\text {per, }}$, is obtained from the original equation (5) set on $\Omega$ by zooming in at the scale $\varepsilon$ and looking at variations around linear responses. The understanding of how $u_{\varepsilon}$ converges to $u^{*}$ in topologies other than $H^{1}$, like in all the Sobolev spaces $W^{k, p}$ or also in Hölder spaces $C^{k, \alpha}$, is obtained by a careful analysis of the Green functions associated to the differential operators $-\operatorname{div}\left(a_{\text {per }} \nabla\right.$. $)$ and $-\operatorname{div}\left(A^{*} \nabla.\right)$ at play in (5) and (6). This was carried out in a series of seminal works [2] by Marco Avellaneda and Fang-Hua Lin in the 1980s and recent works by Carlos Kenig and collaborators. The questions are related to harmonic analysis and the theory of Calderòn-Zygmund operators. All in all, the problem is now well understood theoretically.

The reader familiar with computational science readily understands that the approach provides a natural numerical approximation of $u_{\varepsilon}$.

Homogenization of periodic problems goes far beyond the simple diffusive linear setting described above; see the two reference textbooks by major contributors to the field: A. Bensoussan, J.-L. Lions, and G. Papanicolaou [4] and V. V. Jikov, S. M. Kozlov, and O. A. Oleĭnik [15]. A large portfolio of linear partial differential equations and systems of partial differential equations has been successfully addressed in homogenization problems, along with the associated eigenproblems, time-dependent variants, etc. Many nonlinear problems have also been addressed, should the nonlinearity arise from the terms of the equation themselves or from the geometry of the domain on which the equation is set: linear equation on a perforated domain, on a domain with an unknown or moving boundary, .... State-of-the-art results include elaborate equations 


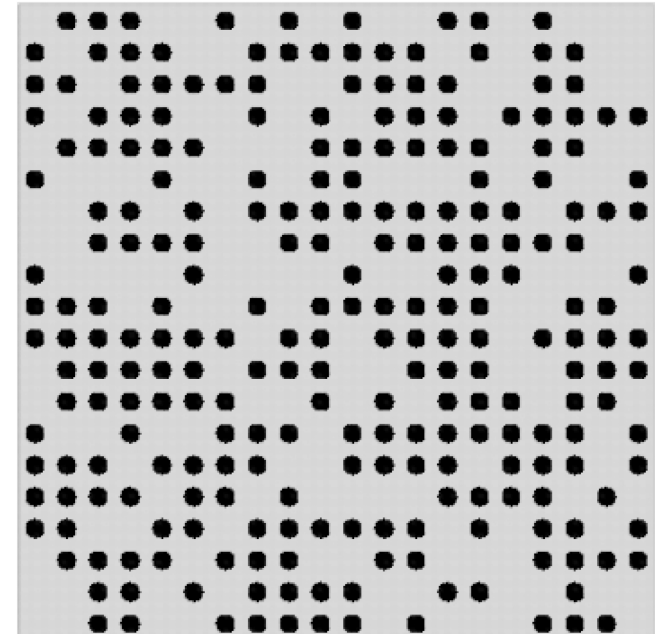

Figure 4. Defects in a periodic structure. In the unperturbed periodic environment, the inclusions are periodic. The elimination of some of these inclusions are the defects considered. The elimination may be deterministic or random. One may also consider small probabilities of elimination and construct the corresponding mathematical setting.

such as fully nonlinear equations, Hamilton-Jacobi type equations, etc. Several important questions remain in the periodic setting, in particular for hyperbolic equations, but the challenges we wish to now return to concern settings other than periodic.

\section{Homogenization Problems with Defects}

We return to the diffusion equation (5) but do not assume any longer that $a_{\varepsilon}$ is a rescaled periodic function $a_{\text {per }}(x / \varepsilon)$. Under quite general and mild assumptions on the (possibly matrix-valued) diffusion coefficient $a_{\varepsilon}$, presumably varying at the tiny scale $\varepsilon$, the equation admits a homogenized limit, which is indeed of the form (6). Celebrated results along these lines are due to Ennio De Giorgi, Sergio Spagnolo, and Luc Tartar and their respective collaborators; see [1, Chapter 1], [20] for reviews. The corresponding theories carry the names of $\Gamma$-limit, $G$-convergence, $H$ convergence. The strength of such results is their generality. They are essentially obtained by a compactness argument. In essence, for our particular setting (5), the sequence of inverse operators $\left[-\operatorname{div}\left(a_{\varepsilon} \nabla .\right)\right]^{-1}$ is weakly compact in the suitable topology, it converges, up to an extraction, and its limit can be proven to be an operator of the same type, namely $\left[-\operatorname{div}\left(A^{*} \nabla .\right)\right]^{-1}$. On the other hand, and precisely because of the generality, not much is known on the limit when $a_{\varepsilon}$ is generic. This contrasts with periodic homogenization which is both explicit-the limit coefficient $A^{*}$ is known by a formula, amenable to computation, as a function of the, also known, corrector-and precise-the rate of convergence of $u_{\varepsilon}$ to $u^{*}$ is known for a large variety of norms. Besides their theoretical interest per se, the above two ingredients together allow for envisioning a practical numerical approach for the computation of the homogenized limit that is certified by a numerical analysis.

The question arises to find sufficiently general settings that still allow for the quality of results of the periodic setting. This brings us back to our question of modeling defects, if defects are seen as violations of periodicity. The recent decade has witnessed several mathematical endeavors in this direction. Definite progress along this line concerns random homogenization. This setting has been originally addressed, in the linear case, in seminal works by George Papanicolaou, Srinivasa Varadhan, and Sergei Kozlov, and, in the nonlinear case, by Gianni Dal Maso and Luciano Modica, and by Luis Caffarelli and Panagiotis Souganidis. Remarkable progress has recently been made in a series of significant works by Felix Otto, Antoine Gloria, Scott Armstrong, Charles Smart, Jean-Christophe Mourrat, and their coworkers; see e.g. [13] for an example of such a contribution. The "partial differential equation" version of our random defects $X_{k}(\omega)=k+Y_{k}(\omega)$, and of much more general random distributions of defects, is now well understood:

$$
-\operatorname{div}\left(a(x / \varepsilon, \omega) \nabla u_{\varepsilon}(x, \omega)\right)=f,
$$

with $a(., \omega)$ ergodic stationary. Most qualitative properties of periodic homogenization are proven to survive in random homogenization, with some differences though (the nature and rates of convergences may be substantially different). Of course, an important point is that these properties are now established on average, that is, mathematically, in expectation, in law, or at best almost surely. Put differently, nothing is known in full generality on a particular realization of the random defects. On the other hand, studies regarding periodic settings perturbed by specific types of defects, again in the spirit of our simple atomistic model above, have been conducted by the author in collaboration with Xavier Blanc and Pierre-Louis Lions [5]. We now give one prototypical example of such a setting, where we illustrate what we believe is the novelty of the mathematical questions involved.

Return to (5) and assume that $a_{\varepsilon}=a(. / \varepsilon)$ where the coefficient $a$ models a periodic material perturbed by a localized defect. This setting, mathematically, may be encoded in $a=a_{\mathrm{per}}+\tilde{a}$ for $\tilde{a} \in L^{p}\left(\mathbb{R}^{n}\right)$ for some $p<+\infty$. The presence of this defect does not affect the macroscopic behavior, that is, the homogenized equation (6). The homogenized coefficient $A^{*}$ remains the same, because it actually depends only on averages of $a$ over large volumes for which the addition of a function such as $\tilde{a}$ does not matter. On the other hand, when it comes to making this limit more precise, one intuitively realizes, zooming in locally in the material, that the corrector equation that describes the microscopic response of the material reads as

$$
-\operatorname{div}\left(a\left(e_{i}+\nabla w_{i}\right)\right)=0,
$$


thus is different from (7). In sharp contrast with (7), the equation (9) does not reduce to an equation set on a bounded domain with periodic boundary conditions. In essence, it is posed on the entire ambient space $\mathbb{R}^{n}$, a reflection of the fact that, at the microscopic scale, the defect has broken the periodicity of the environment: the local response is affected by the defect and depends on the state of the whole material. A considerable mathematical difficulty follows. The classical toolbox for the study of the well-posedness of-here linear-equations on bounded domains, namely the Lax-Milgram lemma in the elliptic case, the Fredholm alternative, etc., all techniques that one way or another rely upon the boundedness of the domain or the compactness of the setting, are now ineffective. Should $a$ be random, then equation (9) would admit an equivalent formulation on the abstract probability space, and this would temporarily make up for compactness, even though other significant complications indeed arise. Here, the difficulty must be embraced. A related difficulty is to define the set of admissible functions for solutions, or the variational space in an energetic formulation of the problem. The decay of these functions away from the defect is key in the mathematical study. In that respect, we recover here a classical difficulty in the more general context of the modeling of defects. For instance, works by John Ball and his collaborators underlined the importance of the selection of the functional variational space in all problems of the calculus of variation and in particular when modeling defects in liquid crystals [3]. In the specific case $a=a_{\mathrm{per}}+\tilde{a}$ with $\tilde{a} \in L^{p}\left(\mathbb{R}^{n}\right)$, one seeks the solution to (9) under the form $w_{i}=w_{p e r, i}+\widetilde{w}_{i}$, that is, with reference to the periodic solution $w_{\text {per }, i}$ to (7) - a trick that, since our first section, the reader is not a stranger to. Equation (9) rewrites as

$$
-\operatorname{div}\left(a \nabla \widetilde{w}_{i}\right)=\operatorname{div}(\widetilde{f})
$$

where $\widetilde{f} \in L^{p}\left(\mathbb{R}^{n}\right)$. This suggests that the suitable functional space for $\nabla \widetilde{w}$ is $L^{p}\left(\mathbb{R}^{n}\right)$. The question then reduces to whether $[\nabla][\operatorname{div}(a \nabla .)]^{-1}[\operatorname{div}]$ operates continuously in $L^{p}\left(\mathbb{R}^{n}\right)$. In the above specific setting, this holds true for all $1<p<+\infty$ (see [5]).

The procedure above is not restricted to the linear diffusion problem (5). One may consider semilinear equations, quasi-linear equations, systems, etc. The study gets all the more delicate as the complexity of the equation increases. In order to show the extent of the complications, we temporarily consider the homogenization of an equation not strictly related to materials, a viscous HamiltonJacobi equation such as

$$
-\varepsilon \Delta u_{\varepsilon}+u_{\varepsilon}+H\left(\nabla u_{\varepsilon}\right)-V(x / \varepsilon)=0,
$$

which leads to a corrector problem of the form

$$
-\Delta w+H(\nabla w)-V(x)=\bar{H},
$$

where for both equations the notion of solution is that of viscosity solutions. For a periodic potential $V$, the problem is now standard, but introducing an additional local defect makes it atypical. The historical contribution on the periodic setting is a landmark article by P.-L. Lions, G. Papanicolaou, and S. R. S. Varadhan, while the random setting was first considered by Fraydoun Rezakhanlou and James Tarver, and by Panagiotis Souganidis. Furthermore, on equations such as (10), we see another feature arising. Equation (5), as a diffusive equation, is in some loose sense "forgiving": a local microscopic defect is forgotten at the macroscopic scale, that is, in the homogenization limit (6). Diffusion smooths things over, and unless one looks at things locally, as in the corrector problem (9), the defect is harmless. In equations of the type (10), where other types of phenomena, such as propagation of waves, or fronts, can be dominant, this is no longer true: waves see defects as obstacles in their propagation, and the solution is modified. A local microscopic defect may affect the macroscopic, homogenized limit. This may already be seen upon considering simple 1-dimensional equations: take $u_{\varepsilon}+\left|\left(u_{\varepsilon}\right)^{\prime}\right|=b(x / \varepsilon)$ for $b$ smooth, compactly supported, nonpositive, and attaining its minimum $b(0)=-1$ at zero; interpret the right-hand side as a defect superimposed to the null function; and check that $u_{\varepsilon}$ converges to $-\exp (-|x|)$, a function macroscopically different from the null function, the unique (bounded) solution, in the viscosity sense, to $u+\left|u^{\prime}\right|=0$.

Various other cases of defects may be considered for homogenization problems that are otherwise "simple." They may formally decay at infinity, like the "localized" functions $\tilde{a}$ manipulated above, or not. In the former case, the problem at infinity (that is, the problem obtained upon translating the equation far away from the defect) is identical to the underlying periodic problem. In the latter case, the situation may sensitively depend upon what the problem "at infinity" - in the same sense as above-looks like. There may even exist several such problems. This is the case for our simple atomistic system (3) above. Whether one sits close to an $X_{p^{2}}$ for $p$ integer, or far away from such points, one does not see the same environment asymptotically. Another prototypical example is related to the modeling of grain boundaries in materials science: two different, periodic structures are connected across an interface. The defect is, say, the plane separating the two structures, and at large distances from this interface, different periodic structures are present, depending upon which side of the interface is considered. The corresponding mathematical problem is theoretically interesting and practically relevant. In all cases, the challenge is to identify the homogenized, macroscopic limit, while, in the meantime, retaining some of the microscopic features that make the problem relevant. 


\section{Related Problems}

It is interesting to notice that the questions we discuss on the corrector equations (7), (9), (11), although arising in the specific context of homogenization, are genuine questions on partial differential equations and have their own interest in the modeling of materials, for a large category of equations set at one given scale. Localization of classical or quantum waves in periodic materials owing to the presence of defects [19] requires the study of eigensystems of the type

$$
-\operatorname{div}\left(\frac{1}{\varepsilon(x)} \nabla u\right)=\lambda u
$$

or

$$
-\Delta u+V(x) u=\lambda u,
$$

respectively, for dielectric constants $\varepsilon(x)$ or potentials $V(x)$ that may be modeled as the diffusion constants $a(x)$ above. As is well known, the spectrum of a periodic operator has a band structure and generalized eigenfunctions that are periodic up to phases. Defects in the periodic background may create eigenvalues associated with localized, nonperiodic, eigenfunctions. The understanding of such phenomena is key for the modeling of material defects at the microscopic scale. The famous problem of Anderson localization, related to the presence of random defects, is yet another example of the theoretical and practical importance of such issues; see [19].

An example of a practically important application consists of the modeling of waveguides, as in the studies conducted by Patrick Joly, Sonia Fliss, and their collaborators; see e.g. [12]. Such problems, and in particular the scattering properties of the device, are sensitive to the periodic structure of the waveguide, and to the possible presence of defects therein. The typical modeling equation reads as

$$
-\Delta u-n^{2}(x) \omega^{2} u=f,
$$

where the refraction index $n$ encodes the nature of the material and may be a perturbation by some defects of an otherwise periodic matrix. Several of the options considered above for modeling various categories of defects can be useful in this particular framework.

In closing, we emphasize that the many options for modeling defects, depending on their nature, geometry, random character, etc., which we have approached above, have a manyfold interest. They provide a selection of interesting problems in the theory of partial differential equations which are all relevant practically and challenge our mathematical understanding of the equations considered. They allow us to explore how sensitively the properties of a given equation depend on the nature of its coefficients. Put formally, we indeed study the linear tangent operator of the map associating the solution to a given coefficient in the equation. There also is, of course, a practical and numerical interest. Our study provides a toolbox that may be useful for a large spectrum of problems in the engineering sciences and prepares the design, the analysis, and the improvements of the numerical approaches. Multiscale materials science is indeed a heavy user of various numerical techniques: multiscale finite elements methods, heterogeneous multiscale method, and many others; see [11]. There is a definite interest in certifying these approaches in a variety of situations, especially when materials have defects. The theoretical analysis that has been overviewed above may constitute a first step toward this goal.

\section{References}

[1] G. Allaire, Shape optimization by the homogenization method, Applied Mathematical Sciences, 146, New York, Springer, 2002. MR1859696

[2] M. Avellaneda and F.-H. Lin, Compactness methods in the theory of homogenization, Parts I \& II, Comm. Pure Appl. Math. 40 (1987), no. 6, 803-847, 42 (1989), no. 2, 139172. MR910954

[3] J. Ball, Liquid crystals and their defects, Mathematical thermodynamics of complex fluids, Lecture Notes in Math., 2200, 1-46, Springer, 2017. MR3729353

[4] A. Bensoussan, J. L. Lions, and G. Papanicolaou, Asymptotic analysis for periodic structures, Studies in Mathematics and its Applications, 5, North-Holland Publishing Co., Amsterdam-New York, 1978. MR503330

[5] X. Blanc, C. Le Bris, and P.-L. Lions, On correctors for linear elliptic homogenization in the presence of local defects, Comm. Partial Differential Equations 43 (2018), no. 6, 965-997. MR3909031

[6] A. Braides and M. S. Gelli, From discrete systems to continuous variational problems: an introduction, Topics on concentration phenomena and problems with multiple scales, Lect. Notes Unione Mat. Ital. 2, Springer, Berlin, 2006, 377. MR2267880

[7] E. Cancès and C. Le Bris, Mathematical modeling of point defects in materials science, M3AS 23 (2013), 1795-1859. MR3078676

[8] P. Cardaliaguet, C. Le Bris, and P. Souganidis, Perturbation problems in homogenization of Hamilton-Jacobi equations, J. Math. Pures Appl. 117 (2018), 221-262. MR384151I

[9] A. V. Cherkaev and R. V. Kohn, Topics in the mathematical modelling of composite materials, reprint of the 1997 edition, Modern Birkhäuser Classics, 2018. MR3822094

[10] G. Dal Maso, A. DeSimone, and F. Tomarelli, Variational problems in material science, Progress in Nonlinear Differential Equations and Their Applications, 68, Birkhäuser, Basel, 2006. MR2222648

[11] B. Engquist and P. E. Souganidis, Asymptotic and numerical homogenization, Acta Numerica (2008), 147-190. MR2436011

[12] S. Fliss and P. Joly, Wave propagation in locally perturbed periodic media (case with absorption): numerical aspects, J. Comput. Phys. 231 (2012), no. 4, 1244-1271. MR2876453

[13] A. Gloria and F. Otto, Quantitative results on the corrector equation in stochastic homogenization, J. Eur. Math. Soc. (JEMS) 19 (2017), no. 11, 3489-3548. MR3713047 


\section{Combinatorial Algebraic}

Geometry

\section{February 1 - May 7, 2021}

\section{ORGANIZING COMMITTEE}

Anders Buch, Rutgers University

Melody Chan, Brown University

June Huh, Princeton University

Thomas Lam, University of Michigan

Leonardo Mihalcea, Virginia Polytechnic Institute

Sam Payne, University of Texas at Austin

Lauren Williams, Harvard University

\section{PROGRAM DESCRIPTION}

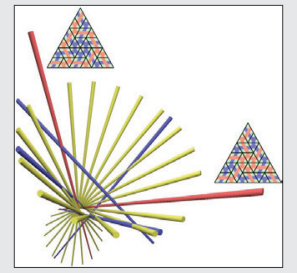

Research in combinatorial algebraic geometry utilizes combinatorial techniques to answer questions about geometry. It also uses geometric methods to provide powerful tools for studying combinatorial objects.

Much research in combinatorial algebraic geometry relies on mathematical software to explore and enumerate combinatorial structures and compute geometric invariants. Writing the required programs is a considerable part of many research projects. The development of new mathematics software is therefore prioritized in the program.

This program will bring together experts in both pure and applied parts of mathematics as well mathematical programmers, all working at the confluence of discrete mathematics and algebraic geometry, with the aim of creating an environment conducive to interdisciplinary collaboration.

\section{icЕ厂ा}

Institute for Computational and Experimental Research in Mathematics

Proposals being accepted: Semester Program

Topical/Hot Topics Workshops

Small Group Research Program

Summer Undergrad Program

ICERM is a National Science Foundation Mathematics Institute at Brown University in Providence, RI.
Appications being accepted: Semester Program or Workshop Postdoctoral Fellowship

Sponsorships being accepted: Academic or Corporate
[14] R. D. James, Materials from mathematics, Bull. Amer. Math. Soc. N.S. 56 (2019), 1-28. MR3886142

[15] V. V. Jikov, S. M. Kozlov, and O. A. Oleĭnik, Homogenization of differential operators and integral functionals, SpringerVerlag, Berlin, 1994. MR1329546

[16] C. Le Bris and P.-L. Lions, From atoms to crystals: a mathematical journey, Bull. Amer. Math. Soc. N.S. 42 (2005), $291-$ 363. MR2149087

[17] T. Lelièvre, M. Rousset, and G. Stoltz, Free energy computations. A mathematical perspective, Imperial College Press, London, 2010. MR2681239

[18] M. Luskin and Ch. Ortner, Atomistic-to-continuumcoupling, Acta Numerica (2013), 397-508. MR3038699

[19] P. Stollmann, Caught by disorder. Bound states in random media, Progress in Mathematical Physics, Vol. 20, 2001. MR1935594

[20] L. Tartar, The general theory of homogenization. A personalized introduction, Lecture Notes of the Unione Matematica Italiana, 7, Springer-Verlag, Berlin; UMI, Bologna, 2009. MR2582099

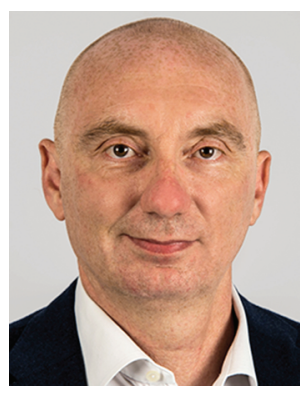

Claude Le Bris

\section{Credits}

Opening image is courtesy of Getty.

Figure 1 is courtesy of Claude Le Bris.

Figure 2 is courtesy of Kai Nordlund. Original publication: Nordlund et al., Nature Communications 9 (2018), 1084. Creative Commons license: creativecommons . org/1icenses/by/4.0/

Figure 3 is courtesy of Alexander Shapeev.

Figure 4 is courtesy of SIAM MMS 9 (2011), no. 2, 513544. ${ }^{\odot 2011 ~ S o c i e t y ~ f o r ~ I n d u s t r i a l ~ a n d ~ A p p l i e d ~ M a t h e m a t-~}$ ics. Reprinted with permission. All rights reserved.

Photo of Claude Le Bris is courtesy of Pierre \& Gilles.

\section{icerm.brown.edu}

